\title{
Epithelial-to-mesenchymal transition in the development and progression of adenocarcinoma and squamous cell carcinoma of the lung
}

\author{
Ludmila Prudkin ${ }^{1}$, Diane D Liu ${ }^{2}$, Natalie C Ozburn ${ }^{3}$, Menghong Sun ${ }^{1}$, Carmen Behrens ${ }^{3}$, \\ Ximing Tang ${ }^{3}$, Kathlynn C Brown ${ }^{4,5}$, B Nebiyou Bekele ${ }^{2}$, Cesar Moran ${ }^{1}$ and \\ Ignacio I Wistuba ${ }^{1,3}$ \\ ${ }^{1}$ Department of Pathology, The University of Texas MD Anderson Cancer Center, Houston, TX, USA; \\ ${ }^{2}$ Department of Biostatistics, The University of Texas MD Anderson Cancer Center, Houston, TX, USA; \\ ${ }^{3}$ Department of Thoracic/Head and Neck Medical Oncology, The University of Texas MD Anderson Cancer \\ Center, Houston, TX, USA; ${ }^{4}$ Division of Translational Research, Department of Internal Medicine, The \\ University of Texas Southwestern Medical Center, Dallas, TX, USA and ${ }^{5}$ Simmons Comprehensive Cancer \\ Center, The University of Texas Southwestern Medical Center, Dallas, TX, USA
}

\begin{abstract}
Epithelial-to-mesenchymal transition is a process in which cells undergo a developmental switch from an epithelial to a mesenchymal phenotype. We investigated the role of this phenomenon in the pathogenesis and progression of adenocarcinoma and squamous cell carcinoma of the lung. Archived tissue from primary tumors $(n=325)$, brain metastases $(n=48)$ and adjacent bronchial epithelial specimens $(n=192)$ were analyzed for immunohistochemical expression by image analysis of E-cadherin, $\mathrm{N}$-cadherin, integrin- $\alpha \mathrm{v} \beta 6$, vimentin, and matrix metalloproteinase-9. The findings were compared with the patients' clinicopathologic features. High expression of the epithelial-to-mesenchymal transition phenotype (low E-cadherin and high $\mathrm{N}$-cadherin, integrin- $\alpha \mathrm{v} \beta 6$, vimentin, and matrix metalloproteinase-9) was found in most lung tumors examined, and the expression pattern varied according to the tumor histologic type. Low E-cadherin membrane and high $\mathrm{N}$-cadherin cytoplasmic expression were significantly more common in squamous cell carcinoma than in adenocarcinoma $(\boldsymbol{P}=\mathbf{0 . 0 0 2}$ and 0.005 , respectively). Dysplastic lesions had significantly lower expression of the epithelial-to-mesenchymal transition phenotype than the squamous cell carcinomas, and integrin- $\alpha \mathrm{V} \beta 6$ membrane expression increased stepwise according to the histopathologic severity. Brain metastases had decreased epithelial-to-mesenchymal transition expression compared with primary tumors. Brain metastases had significantly lower integrin- $\alpha \mathrm{V} / 6$ membrane $(P=0.04), \mathrm{N}$-cadherin membrane, and cytoplasm $(P<0.0002)$ expression than the primary tumors. The epithelial-to-mesenchymal transition phenotype is commonly expressed in primary squamous cell carcinoma and adenocarcinoma of the lung; this expression occurs early in the pathogenesis of squamous cell carcinoma. Brain metastases showed characteristics of reversed mesenchymal-to-epithelial transition. Our findings suggest that epithelial-to-mesenchymal transition is a potential target for lung cancer chemoprevention and therapy.
\end{abstract}

Modern Pathology (2009) 22, 668-678; doi:10.1038/modpathol.2009.19; published online 6 March 2009

Keywords: epithelial-to-mesenchymal transition; tissue microarray; immunohistochemical analysis; lung cancer; preneoplasia; brain metastases

Lung cancer remains the leading cause of cancerrelated death in the United States. ${ }^{1}$ It is a highly complex neoplasm ${ }^{2}$ that includes several histologic

Correspondence: Dr II Wistuba, Department of Pathology, Unit 85, The University of Texas MD Anderson Cancer Center, 1515 Holcombe Blvd., Houston, TX 77030-4009, USA.

E-mail: iiwistuba@mdanderson.org

Received 15 October 2008; revised 7 February 2009; accepted 9 February 2009; published online 6 March 2009 types, the most common being small-cell lung carcinoma and two types of non-small-cell lung carcinomas, adenocarcinoma and squamous cell carcinoma. ${ }^{3}$ Despite therapeutic advances, the 5 -year survival rate across all stages of the disease is approximately $15 \%,{ }^{1}$ as many patients are not diagnosed until the advance stage of the disease. Surgery is considered the best treatment option, but only approximately $25 \%$ of lung tumors with adenocarcinoma and squamous cell carcinoma 
histology are suitable for potentially curative resection. ${ }^{4}$ Lung tumors are believed to result from a progressive series of lesions. ${ }^{5}$ This sequence has been clearly established in squamous cell carcinoma, but in adenocarcinoma, the progression sequence is less clear. ${ }^{5} \mathrm{~A}$ deeper understanding of the molecular mechanisms involved in the pathogenesis and progression of lung cancer may lead to new and more effective strategies for early detection and targeted chemoprevention and treatment.

Epithelial-to-mesenchymal transition is a process in which cells undergo a developmental switch from an epithelial to a motile mesenchymal phenotype. ${ }^{6}$ This process has been related to embryologic morphogenesis, fibrosis, and lately, to the progression and metastasis of epithelial tumors. ${ }^{7}$ Solid tumor progression requires epithelial-to-mesenchymal transition for tumor cells to invade and metastasize. Colon, prostate, and breast cancer are tumors with the epithelial-to-mesenchymal transition phenotype. ${ }^{8}$ In lung cancer, this phenotype has been studied in vitro; the expression of individual markers in this transition has been described, and these markers are associated with prognosis., ${ }^{9,10}$ These findings have led to the hypothesis that epithelial-to-mesenchymal transition is a target for lung cancer therapy. In addition, data suggest that this phenotype is influenced by epidermal growth factor receptor (EGFR) activity and predicts EGFR tyrosine kinase inhibitor sensitivity. ${ }^{9,11}$

To better understand the importance of epithelialto-mesenchymal transition in the pathogenesis and progression of adenocarcinoma and squamous cell carcinoma of the lung, we determined the immunohistochemical expression of five markers related to this phenomenon (E-cadherin, N-cadherin, integrin$\alpha \mathrm{v} \beta 6$, vimentin, and matrix metalloproteinase (MMP)-9) by image analysis of tissue microarray specimens. We compared these findings with patients' clinicopathologic features and the immunohistochemical expression of EGFR and phosphorylated EGFR. In a subset of adenocarcinomas, we compared epithelial-to-mesenchymal transition expression with the presence of EGFR mutations. In addition, we investigated the role of this phenomenon in the early pathogenesis of lung cancer by studying marker expression in the preneoplastic sequence of squamous cell carcinoma. Mesenchymal-to-epithelial transition has been hypothesized to occur at the site of tumor metastasis. ${ }^{6,8}$ Therefore, to determine whether this reverse transition was present in advanced lung tumors, we also analyzed our five markers in lung cancer brain metastases.

\section{Materials and methods}

\section{Case Selection and Tissue Microarray Construction}

We obtained archived, formalin-fixed, paraffin-embedded tissue from surgically resected lung cancer specimens (lobectomies and pneumonectomies) containing tumor and adjacent normal and abnormal epithelial tissues from the Lung Cancer Specialized Program of Research Excellence Tissue Bank at The University of Texas MD Anderson Cancer Center (Houston, TX, USA), which has been approved by the institutional review board. The tissue had been collected from 1997 to 2001 . The tissue specimens were histologically examined and classified using the 2004 World Health Organization classification system. ${ }^{3}$ We selected 325 primary lung tumor tissue samples, including 209 adenocarcinomas and 116 squamous cell carcinomas, for our tissue microarrays. These arrays were constructed using triplicate $1-\mathrm{mm}$ diameter cores per tumor; each core included central, intermediate, and peripheral tumor tissue. Detailed clinical and pathologic information, including demographics, smoking history (never- and ever smokers), and smoking status (never, former, and current), clinical and pathologic TNM stage, overall survival duration, and time to recurrence, was available for most cases (Table 1). Tumors were pathologic TNM stages I-IV according to the revised International System for Staging Lung Cancer. ${ }^{12}$ The expression of integrin $\alpha \mathrm{v} \beta 6$ in the same set of tumors has been previously described. ${ }^{13}$

To assess the immunohistochemical expression of epithelial-to-mesenchymal transition markers in the early pathogenesis of squamous cell carcinoma of the lung, we studied tissue microarrays containing 192 bronchial epithelium specimens (normal histology, $N=49$; basal cell hyperplasia, $N=76$;

Table 1 Demographic and clinicopathologic data by histologic tumor type

\begin{tabular}{lrcc}
\hline Characteristic & $\begin{array}{c}\text { Total } \\
(\mathrm{N}=325)\end{array}$ & $\begin{array}{c}\text { Adenocarcinoma } \\
(\mathrm{N}=209)\end{array}$ & $\begin{array}{c}\text { Squamous } \\
\text { cell } \\
\text { carcinoma } \\
(\mathrm{N}=116)\end{array}$ \\
& & & \\
Sex & & 128 & 45 \\
Female & 173 & 81 & 71 \\
Male & 152 & & \\
Stage & & 139 & 66 \\
I & 205 & 28 & 34 \\
II & 62 & 75 & 14 \\
III & 49 & & 2 \\
IV & 9 & 152 & \\
Smoking history & & 57 & 111 \\
Yes & 263 & & 4 \\
No & 61 & 57 & 4 \\
& & 92 & 69 \\
Smoking status & & \\
Never & & 60 & 42 \\
Former & 61 & & \\
Current & 161 & & \\
\hline
\end{tabular}

${ }^{\mathrm{a}}$ Smoking history and status was not available for one squamous cell carcinoma patient. 
squamous metaplasia, $N=13$; squamous dysplasia, $N=34$; and carcinoma in situ, $N=20$ ) from 89 patients with lung cancer. For statistical analysis, we grouped the lesions by tissue type: normal and reactive (including normal, hyperplasia, and squamous metaplasia) and dysplastic (including dysplasia and carcinoma in situ). Finally, to determine the expression of epithelial-to-mesenchymal transition markers in lung cancer metastasis, we examined tissue microarrays containing 48 brain metastases, including 37 from adenocarcinomas and 11 from squamous cell carcinomas.

\section{Immunohistochemical Staining and Evaluation}

Antibodies against the following molecules were purchased and used: E-cadherin (Santa Cruz Biotechnology, Santa Cruz, CA, USA; catalogue number Sc-8426; dilution 1:100), N-cadherin (Zymed, Carlsbad, CA, USA; catalogue number 18-0224, concentration 1:100), integrin- $\alpha \mathrm{v} \beta 6$ (Calbiochem, Gibbstown, NJ, USA; catalogue number 407317; dilution 1:300), vimentin (Dako, Carpinteria, CA, USA; catalogue number M-0725; dilution 1:400), MMP-9 (Genetex, San Antonio, TX, USA; catalogue number GTX-58899; dilution 1:400), EGFR, and phosphorylated EGFR (Tyr-1086; Invitrogen, Carlsbad, CA, USA; catalogue numbers 28-0005 and 36-9700, respectively; dilution 1:100). Immunohistochemical staining was performed as follows: 5 - $\mu$-thick sections from formalin-fixed and paraffinembedded tissue were deparaffinized, hydrated, heated in a steamer for $10 \mathrm{~min}$ with $10 \mathrm{mM}$ sodium citrate ( $\mathrm{pH}$ 6.0) for antigen retrieval, and washed in Tris buffer. Peroxide blocking was performed with $3 \% \mathrm{H}_{2} \mathrm{O}_{2}$ in methanol at ambient temperature for $15 \mathrm{~min}$, followed by $10 \%$ bovine serum albumin in Tris-buffered saline-t at ambient temperature for $30 \mathrm{~min}$. The slides were incubated with primary antibody at ambient temperature and washed with phosphate-buffered saline, followed by incubation with biotin-labeled secondary antibody for $30 \mathrm{~min}$. Finally, the samples were incubated with a 1:40 solution of streptavidin-peroxidase for $30 \mathrm{~min}$. Staining was developed with $0.05 \% 3^{\prime}, 3$-diaminobenzidine tetrahydrochloride, which had been freshly prepared in $0.05 \mathrm{~mol} / \mathrm{l}$ Tris buffer at $\mathrm{pH} 7.6$ containing $0.024 \% \mathrm{H} 2 \mathrm{O} 2$ and then counterstained with hematoxylin, dehydrated, and mounted. Lung cancer tumor cell lines pellets and tissues with normal bronchial epithelia were used as the positive control. As the negative control, we used the same specimens used as positive controls but replaced the primary antibody with phosphate-buffered saline.

Protein expression was quantified using the ARIOL image analysis system (Applied Imaging, San Jose, CA, USA). This system gives an immunohistochemistry reading that avoids interobserver and intraobserver variability. For each marker, different cell sites were evaluated: membrane in E-cadherin and integrin- $\alpha \mathrm{v} \beta 6$; membrane and cytoplasm in N-cadherin; and cytoplasm in vimentin and MMP-9. In addition, EGFR and phosphorylated EGFR (Tyr-1086) membranous staining was evaluated. Membrane expression was scored on the basis of the intensity and completeness of immunostaining, similar to Her2-Neu assessment classification. ${ }^{14}$ For the statistical analysis, we used class score, grouped into two categories: 0 and 1, low expression; and, 2 and 3, high expression. Cytoplasm staining was scored to account for both the intensity and extent of protein expression. These scores were used in the statistical analysis of cytoplasm expression. Vimentin is a special marker that is highly expressed in stromal and inflammatory cells, but it is not suitable for image analysis. Therefore, this marker was scored by a trained pathologist (LP) using light microscopy, a four-value intensity scale $(0-3+)$, and percentage extent $(0-100 \%)$. The vimentin score was calculated by multiplying both parameters (range, 0-300).

\section{EGFR Mutation Analysis}

Exons 18 through 21 of EGFR were polymerase chain reaction (PCR)-amplified using intron-based primers as previously described. ${ }^{15,16}$ Approximately 1,000 microdissected cells were used for every PCR amplification. All PCR products were directly sequenced using the Applied Biosystems PRISM dye terminator cycle sequencing method. All sequence variants were confirmed by independent PCR amplifications from at least two independent microdissections and DNA extraction and sequenced in both directions, as previously reported. ${ }^{15,16}$

\section{Statistical Analysis}

The data were summarized using standard descriptive statistics and frequency tabulation. Associations between categorical variables were assessed via crosstabulation, the $\chi^{2}$-test, and Fisher's exact test. The Kruskal-Wallis and Wilcoxon rank-sum tests were performed to determine the differences in continuous variables by clinicopathologic feature. Survival curves were estimated using the Kaplan-Meier method. Univariate and multivariate Cox proportional hazard models were used to assess the effect of covariates on overall survival and recurrence-free survival. All computations were carried out using SAS (Cary, NC, USA) and S-plus 2000 (Cambridge, MA, USA) software. $P$-values less than 0.05 were considered as statistically significant.

\section{Results}

\section{Immunohistochemical Expression of Epithelial-to-Mesenchymal Transition Markers in Lung Cancer Specimens}

Overall, adenocarcinoma and squamous cell carcinoma had high levels of the epithelial-to-mesenchymal 
transition phenotype on the basis of protein expression of the five markers examined (Figure 1; Table 2). Both histologic types had significantly reduced levels of E-cadherin membrane expression, as measured by membrane class scores, compared with corresponding adjacent normal and reactive respiratory epithelium (Figure 2). In contrast, lung tumors had significantly higher cytoplasm scores of $\mathrm{N}$-cadherin and MMP-9 and membrane class scores of N-cadherin and integrin- $\alpha \mathrm{v} \beta 6$ compared with adjacent respiratory epithelium (Figure 2). Vimentin cytoplasm scores were significantly higher in adenocarcinoma tumors than in normal and reactive epithelium but not in squamous cell carcinomas (Figure 2).

Both tumor histologic types had varied patterns of immunohistochemical epithelial-to-mesenchymal transition marker expression (Table 2). E-cadherin membrane expression was low (classes 0 and 1) in $63 \%$ of tumors, and this low level of expression was significantly more common in squamous cell carcinomas $(73 \%)$ than in adenocarcinomas (57\%; $P=0.005$ ). High levels (classes 2 and 3 ) of membrane expression of $\mathrm{N}$-cadherin and integrin- $\alpha \mathrm{v} \beta 6$ were detected in 68 and $56 \%$ of lung cancers, with no differences by histologic type (Table 2). However, the $\mathrm{N}$-cadherin cytoplasm score was significantly $(P=0.002)$ higher in squamous cell carcinoma. No significant differences in the level of cytoplasm expression of vimentin and MMP-9 were detected between adenocarcinoma and squamous cell carcinoma.
We compared membrane class scores and cytoplasm scores with patients' demographic and clinicopathologic characteristics, including age, sex, smoking history, and TNM pathologic stage. Interestingly, $\mathrm{N}$-cadherin membrane class and cytoplasm scores were significantly higher $(P=0.004$ and $P=0.0004$, respectively) in ever smokers than in never smokers. No significant association was found between any marker expression and pathologic disease stage. We also determined the prognostic (recurrence-free and overall survival) effect of epithelial-to-mesenchymal transition marker expression; the only marker that was associated with outcome was integrin- $\alpha \mathrm{v} \beta 6$ membrane expression. As stated above, we previously found ${ }^{13}$ that membrane integrin- $\alpha v \beta 6$ is frequently overexpressed in the same set of lung tumor specimens using the same tissue microarray set reported here and that this overexpression significantly affected overall survival (data not shown).

To determine whether there was an association between epithelial-to-mesenchymal transition phenotype and EGFR protein expression in lung tumors, we compared the immunohistochemical expression of our five markers with EGFR and phosphorylated EGFR (Tyr-1086) expression in 225 tumors. No association was detected between the immunohistochemical expression of epithelial-to-mesenchymal transition and the EGFR proteins evaluated. Likewise, tumors' EGFR mutation status was not associated with our five-marker expression in a subset of 135 adenocarcinomas, including 32 (24\%) mutant cases.
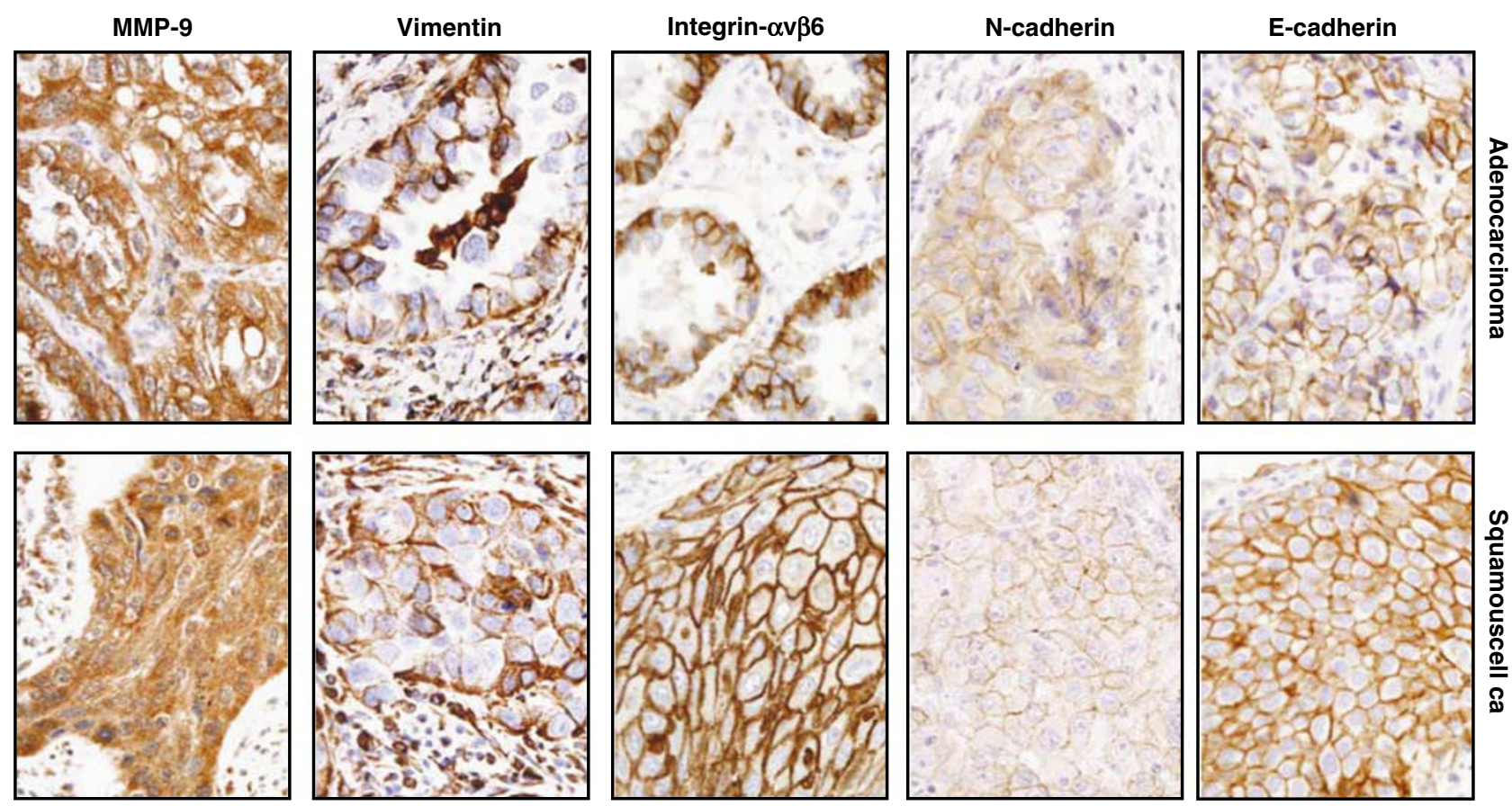

Figure 1 Representative microphotographs of immunohistochemical expression of epithelial-to-mesenchymal transition markers in tissue specimens of primary adenocarcinoma and squamous cell carcinoma. Tumor cells show membrane immunostaining for E-cadherin and integrin- $\alpha \mathrm{v} \beta 6$, membrane and cytoplasm staining for N-cadherin, and cytoplasm staining for vimentin and MMP-9. 
Table 2 Immunohistochemical expression of epithelial-to-mesenchymal transition markers by tumor histologic type

\begin{tabular}{|c|c|c|c|c|}
\hline Marker & Adenocarcinoma & $\begin{array}{l}\text { Squamous cell } \\
\text { carcinoma }\end{array}$ & $\mathrm{P}$-value & Total \\
\hline \multicolumn{5}{|l|}{ E-cadherin } \\
\hline \multicolumn{5}{|l|}{ Membrane class } \\
\hline$N$ & 191 & 110 & \multirow{3}{*}{0.005} & 301 \\
\hline 0 or $1(\%)$ & $108(57)$ & $80(73)$ & & $188(62)$ \\
\hline 2 or $3(\%)$ & $83(43)$ & $30(27)$ & & $113(38)$ \\
\hline \multicolumn{5}{|l|}{$N$-cadherin } \\
\hline \multicolumn{5}{|l|}{ Membrane class } \\
\hline$N$ & 193 & 106 & \multirow{3}{*}{0.73} & 299 \\
\hline 0 or $1(\%)$ & $60(31)$ & 35 (33) & & $95(32)$ \\
\hline 2 or $3(\%)$ & $133(69)$ & $71(67)$ & & $204(68)$ \\
\hline Mean cytoplasm score (s.d.) & $46.4(4.2)$ & $47.8(2.4)$ & 0.002 & $46.9(3.7)$ \\
\hline \multicolumn{5}{|l|}{ Integrin- $\alpha v \beta 6$} \\
\hline \multicolumn{5}{|l|}{ Membrane class } \\
\hline$N$ & 177 & 106 & \multirow[t]{3}{*}{0.89} & 283 \\
\hline 0 or $1(\%)$ & $77(44)$ & $47(44)$ & & $124(44)$ \\
\hline 2 or $3(\%)$ & $100(56)$ & $59(56)$ & & $159(56)$ \\
\hline \multicolumn{5}{|l|}{ Vimentin } \\
\hline \multicolumn{5}{|l|}{ Cytoplasm score } \\
\hline$N$ & 172 & 90 & \multirow[b]{2}{*}{0.15} & 262 \\
\hline Mean (s.d.) & $62.1(92.6)$ & $42.2(72.4)$ & & $55.3(86.6)$ \\
\hline \multicolumn{5}{|l|}{$M M P-9$} \\
\hline \multicolumn{5}{|l|}{ Cytoplasm score } \\
\hline$N$ & 179 & 96 & & 275 \\
\hline Mean (s.d.) & $44.8(9.9)$ & $44.9(8.4)$ & 0.95 & $44.8(9.4)$ \\
\hline
\end{tabular}

MMP-9, metalloproteinase-9; s.d., standard deviation.

\section{Epithelial-to-Mesenchymal Transition Marker Expression in the Sequential Pathogenesis of Squamous Cell Carcinoma}

We determined the expression of our five markers in 192 epithelial specimens containing histologically normal, hyperplastic, squamous metaplastic, or squamous dysplastic bronchial epithelia adjacent to squamous cell carcinomas obtained from 89 patients. We found an increased level of the epithelial-to-mesenchymal transition phenotype, corresponding to increased histopathologic severity, in squamous preneoplasias (dysplasias and carcinoma in situ) compared with corresponding primary tumors. The membrane expression of E-cadherin was significantly reduced in tumors compared with preneoplastic lesions (Figure 3), but the membrane expression of $\mathrm{N}$-cadherin and integrin- $\alpha \mathrm{v} \beta 6$ (Figure 3) and cytoplasm expression of N-cadherin and vimentin (data not shown) were significantly increased. Although squamous preneoplasias had significantly higher expression than the normal and reactive epithelia of membrane integrin- $\alpha \mathrm{v} \beta 6$ (Figure 3) and cytoplasm MMP-9 (data not shown), we found that preneoplasias had significantly lower expression of $\mathrm{N}$-cadherin and vimentin than the normal and reactive epithelia (data not shown). Of interest, integrin- $\alpha \mathrm{v} \beta 6$ membrane expression showed a clear stepwise increase, according to the severity of histopathologic lesions. A high level of expression (classes 2 and 3 ) was detected in 14 of 73
(19\%) normal and reactive epithelia, 18 of $44(41 \%)$ squamous preneoplasias, and 59 of 106 (56\%) primary squamous cell carcinomas.

\section{Immunohistochemical Expression of Epithelial-to-Mesenchymal Transition Markers in Brain Metastases}

We studied the expression of these five markers in 46 lung cancer brain metastases, including 35 adenocarcinomas and 11 squamous cell carcinomas. Interestingly, we found that metastatic sites had a lower level of the epithelial-to-mesenchymal transition phenotype than the primary tumors, which has been suggested to correspond to mesenchymal-toepithelial transition (Table 3). ${ }^{6}$ Squamous cell carcinoma brain metastases had a significantly higher E-cadherin membrane class score than the primary tumors. The metastatic sites of both histologic types had significantly lower integrin- $\alpha \mathrm{v} \beta 6$ membrane and N-cadherin membrane and cytoplasm expression scores than the primary tumors (Table 3).

\section{Discussion}

To our knowledge, this is the first study to fully characterize the expression of multiple epithelial-to-mesenchymal transition markers in lung 
E-cadherin
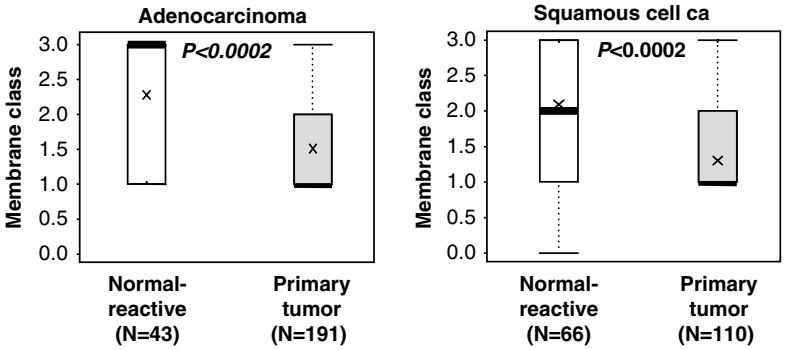

$\mathrm{N}$-cadherin
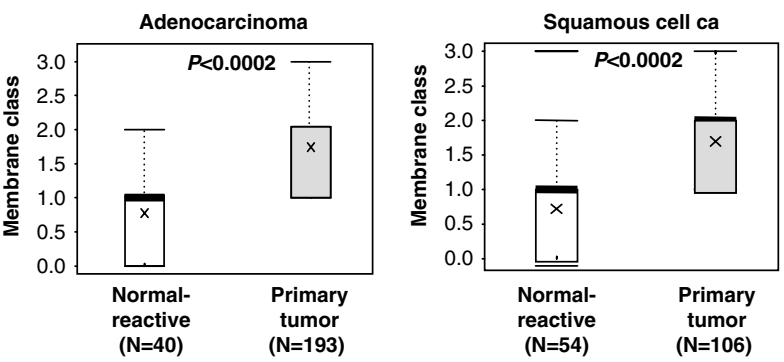

N-cadherin
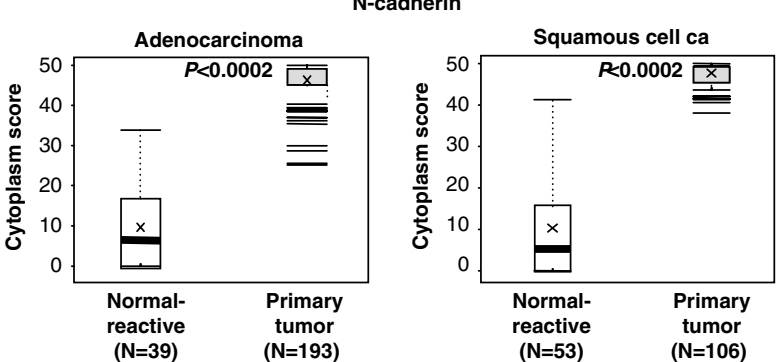

Integrin- $\alpha v \beta 6$
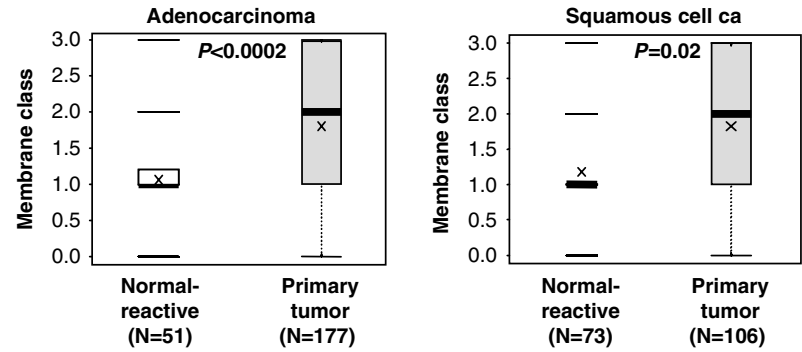

Vimentin
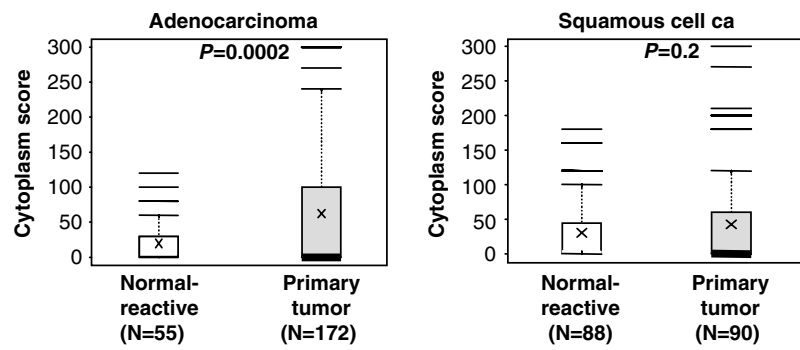

MMP-9
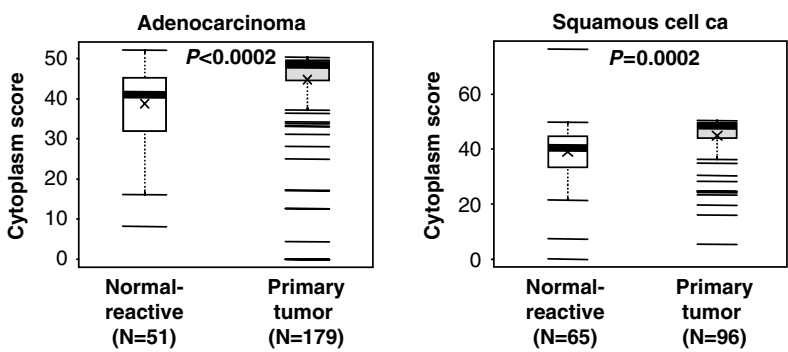

Figure 2 Scores of membrane and cytoplasm immunohistochemical expression of epithelial-to-mesenchymal transition markers in normal and reactive epithelia compared with those in primary tumors. The number of samples is indicated for each histologic group and marker. $P$-values comparing normal epithelial and tumor histologic types are shown for all comparisons.

cancer primary tumors and metastases and the pathogenesis of squamous cell carcinoma. The use of tissue microarrays to assess tumor and epithelial tissue specimens with annotated clinical information, coupled with image analysis of immunohistochemical expression, allowed us to characterize the expression of five epithelial-to-mesenchymal transition markers in a large set of tumor and epithelial specimens comprising the entire spectrum of lung cancer pathogenesis and progression.

First, we demonstrated that adenocarcinoma and squamous cell carcinoma had high levels of the epithelial-to-mesenchymal transition phenotype compared with normal bronchial epithelium. This phenotype is characterized by reduced expression of E-cadherin and overexpression of $\mathrm{N}$-cadherin, integrin- $\alpha \mathrm{v} \beta 6$, MMP-9, and vimentin. The differential expression of this phenotype between normal and tumor tissues demonstrates that this phenomenon is associated with the development of these tumor types. The reduced expression of E-cadherin ${ }^{10,17,18}$ and overexpression of $\mathrm{N}$-cadherin, ${ }^{19}$ integrin- $\alpha \mathrm{v} \beta 6,{ }^{20}$ and MMP- $9^{21-23}$ has been previously reported in tissue specimens from adenocarcinomas and squamous cell carcinoma of the lung. Vimentin is considered a hallmark for the mesenchymal differentiation of cells, and its expression in lung cancer cell lines has been previously associated with other changes related to epithelial-to-mesenchymal transition. ${ }^{9}$ However, to our knowledge, our study is the first to report the expression of multiple of these transition markers in a large set of lung cancers using image analysis assessment of immunostaining.

Tumors are defined by their invasive and metastatic potential. This potential may be achieved by tumor cells via epithelial-to-mesenchymal transition, and multiple histologic, molecular, and transcriptional changes related to the development of this phenotype occur during carcinoma progression. ${ }^{24}$ This process is characterized by morphologic modifications, including epithelial polarized cells acquiring a motile nonpolarized appearance, and molecular changes, including altered expression of growth and transcription factors and modified expression of cytoskeletal and adhesion molecules. ${ }^{6}$ The epithelial-to-mesenchymal transition process has been described extensively for several types of 


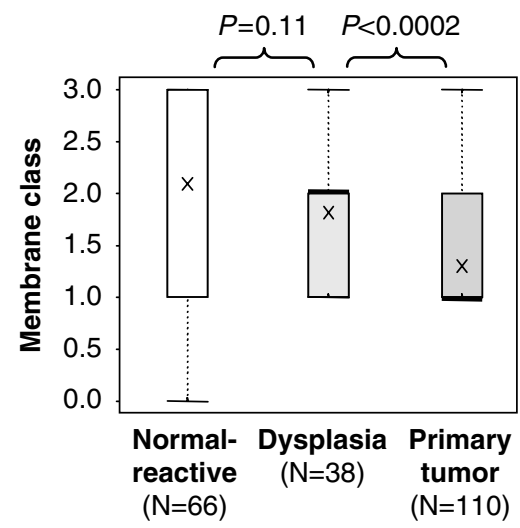

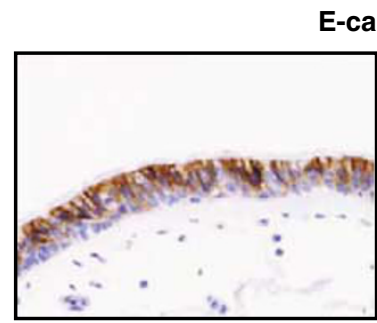

Normal epithelium
E-cadherin

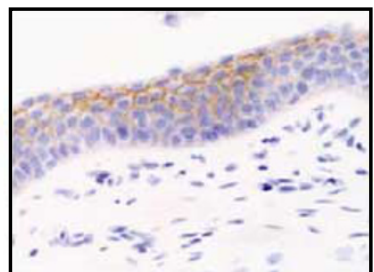

Dysplasia

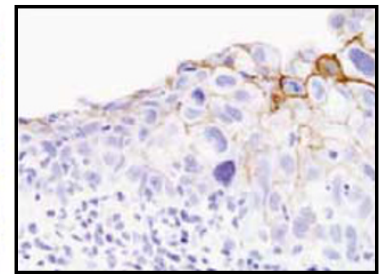

Carcinomain situ

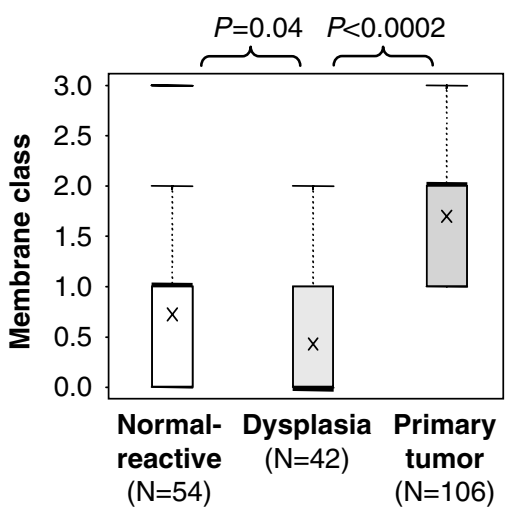

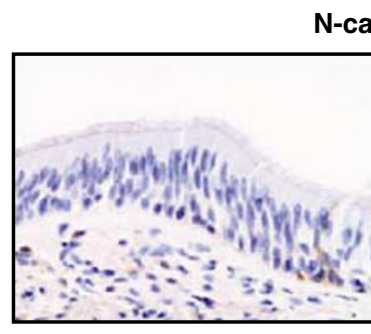

Normal epithelium

\section{-cadherin}

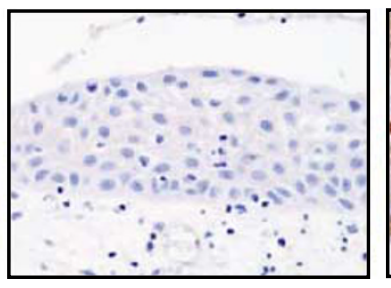

Dysplasia

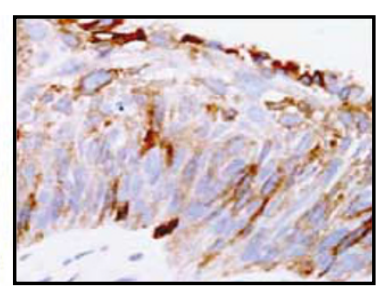

Carcinomain situ

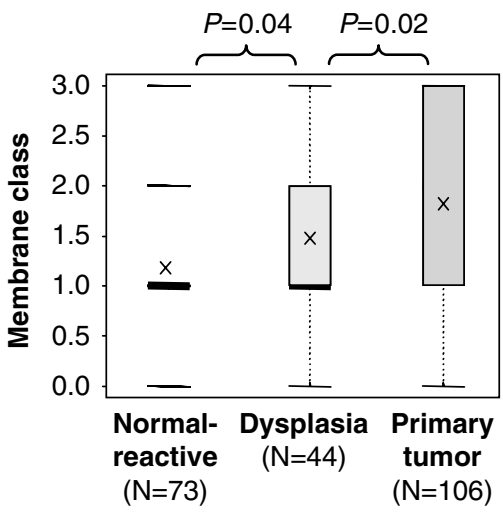

Integrin- $\alpha v \beta 6$

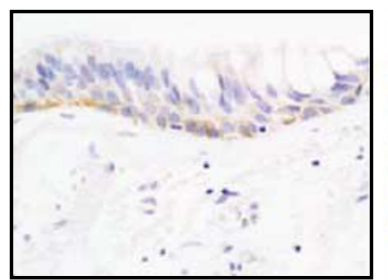

Normal epithelium

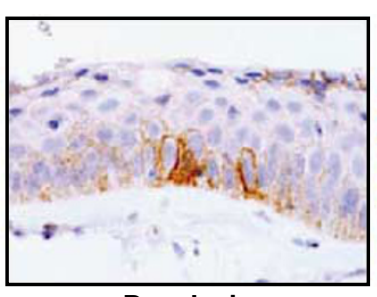

Dysplasia

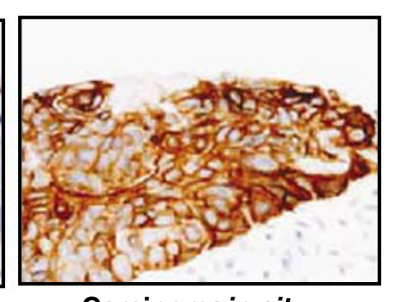

Carcinomain situ

Figure 3 Scores for membrane immunohistochemical expression of E-cadherin, N-cadherin, and integrin- $\alpha \mathrm{v} \beta 6$ in bronchial respiratory epithelial lesions related to the pathogenesis of squamous cell carcinoma of the lung: normal and reactive epithelium, squamous preneoplasia (dysplasias and carcinoma in situ), and primary squamous cell carcinoma. The number of samples is indicated for each histologic group and marker. Significant $P$-values for comparisons between each group are shown. For each marker, representative microphotographs of immunohistochemical expression in tissue specimens of bronchial epithelium with normal, squamous dysplasia, and carcinoma in situ are shown. For E-cadherin, decreased membrane immunostaining was found with increased histologic severity. The opposite phenomenon is shown for cytoplasm N-cadherin and membrane integrin- $\alpha \mathrm{v} \beta 6$ expression.

cancer, particularly colon, breast, and prostate cancer. ${ }^{8,25-28}$ In lung cancer, the loss or switch of adhesion molecules ${ }^{10,29}$ and the expression of classically mesenchymal proteins have been associated with the induction of mesenchymal changes and carcinoma aggressiveness. ${ }^{9}$ In addition, the expression of several growth factors and their effectors, ${ }^{30}$ transcription factors, ${ }^{31,32}$ and proteases $^{33,34}$ have been associated with epithelial-tomesenchymal transition.
Loss of E-cadherin membrane expression and the subsequent reduction of the cells' ability to form stable cell-cell contacts is a hallmarks of epithelial-to-mesenchymal transition. ${ }^{6}$ In lung cancer, E-cadherin downregulation occurs mostly via epigenetic mechanisms, including gene promoter hypermethylation. ${ }^{35} \mathrm{~N}$-cadherin is mainly expressed in nervous system and mesenchymal normal cells. In epithelial tumors, increased N-cadherin immunohistochemical expression (often de novo 
Table 3 Immunohistochemical expression of epithelial-to-mesenchymal transition markers in primary tumors and brain metastases by histologic type

\begin{tabular}{|c|c|c|c|c|c|c|}
\hline \multirow[t]{2}{*}{ Marker } & \multicolumn{3}{|c|}{ Adenocarcinoma } & \multicolumn{3}{|c|}{ Squamous cell carcinoma } \\
\hline & Primary tumor & Metastasis & P-value & Primary tumor & Metastasis & $\mathrm{P}$-value \\
\hline \multicolumn{7}{|l|}{ E-cadherin } \\
\hline \multicolumn{7}{|c|}{ Membrane class } \\
\hline & 191 & 35 & & 110 & 11 & \\
\hline Mean (s.d.) & $1.5(0.6)$ & $1.6(0.7)$ & 0.16 & $1.3(0.5)$ & $1.6(0.8)$ & 0.02 \\
\hline \multicolumn{7}{|l|}{$N$-cadherin } \\
\hline \multicolumn{7}{|l|}{ Membrane class } \\
\hline$N$ & 193 & 37 & & 106 & 11 & \\
\hline Mean (s.d.) & $1.7(0.6)$ & $1.2(0.9)$ & $<0.0002$ & $1.7(0.5)$ & $1(0.4)$ & $<0.0002$ \\
\hline \multicolumn{7}{|c|}{ Cytoplasm score } \\
\hline Mean (s.d.) & $46.4(4.2)$ & $20.5(20.1)$ & $<0.0002$ & $47.8(2.4)$ & $28.6(20.7)$ & $<0.0002$ \\
\hline \multicolumn{7}{|l|}{ Integrin- $\alpha v \beta 6$} \\
\hline \multicolumn{7}{|l|}{ Membrane class } \\
\hline$N$ & 177 & 32 & & 106 & 11 & \\
\hline Mean (s.d.) & $1.8(0.9)$ & $1.2(0.9)$ & $<0.0002$ & $1.8(0.9)$ & $1.5(0.9)$ & 0.04 \\
\hline \multicolumn{7}{|c|}{ Vimentin } \\
\hline \multicolumn{7}{|l|}{ Cytoplasm score } \\
\hline & 172 & 32 & & 90 & 10 & \\
\hline Mean (s.d.) & $62.1(92.6)$ & $61.3(101.3)$ & 0.9 & $42.2(72.4)$ & $15(30.6)$ & 0.04 \\
\hline \multicolumn{7}{|l|}{$M M P-9$} \\
\hline \multicolumn{7}{|c|}{ Cytoplasm score } \\
\hline$N$ & 179 & 31 & & 96 & 8 & \\
\hline Mean (s.d.) & $44.8(9.9)$ & $47.1(2.9)$ & 0.07 & $44.9(8.4)$ & $47.4(1.6)$ & 0.12 \\
\hline
\end{tabular}

MMP-9, metalloproteinase-9; s.d., standard deviation.

expression) is accompanied by E-cadherin downregulation. ${ }^{36}$ In adenocarcinoma and squamous cell carcinoma of the lung, increased $\mathrm{N}$-cadherin membrane and cytoplasm expression has been previously described in approximately one-third of tumors. ${ }^{19}$ Integrin- $\alpha \mathrm{v} \beta 6$, an integrin transmembrane glycoprotein, is integrated across the plasma membrane and provides a link between the extracellular matrix and cytoskeletal molecules. ${ }^{37}$ Integrin- $\alpha \mathrm{v} \beta 6$ overexpression has been found in other epithelial tumors, including colorectal, ${ }^{25}$ ovarian, ${ }^{38}$ and head and neck carcinomas. ${ }^{39}$ Of interest, integrin- $\alpha \mathrm{v} \beta 6$ was recently identified by biopanning peptides in lung cancer cell lines, ${ }^{20}$ and we have reported immunohistochemistry tissue microarray data that validate this method. ${ }^{20}$ MMPs are part of the proteolytic cascade that degrades the extracellular matrix and allows the migration of tumor and endothelial cells. ${ }^{40}$ MMP-9 is a gelatinase capable of forming gaps in the basement membrane to facilitate invasion and metastasis. ${ }^{40}$ High levels of MMP-9 immunohistochemical expression have been reported in nearly $60 \%$ of lung tumors. . $^{21,23,41}$

Our findings indicate that the epithelial-tomesenchymal transition phenotype is homogeneously present in lung tumors with adenocarcinoma and squamous cell carcinoma histology; this phenotype is associated with relatively few clinicopathologic features, including tumor histologic characteristics and patients' smoking status. Squamous cell carcinoma had reduced E-cadherin expression and increased N-cadherin cytoplasmic expression compared with adenocarcinoma histology. To our knowledge, only reduced E-cadherin expression in lung squamous cell carcinoma histology has been previously reported. ${ }^{42,43}$ Interestingly, in our study, the N-cadherin membrane and cytoplasm scores were significantly higher in ever smokers than in never smokers. The biologic reason for this phenomenon is not known. Several studies have consistently found an association between loss of E-cadherin expression and poor prognosis in lung cancer patients treated with surgery with curative intent. $^{10,17,29,43}$ To our knowledge, only one study showed that N-cadherin overexpression was associated with poor outcome in patients with lung tumor with non-small-cell lung cancer types (in a univariate analysis). ${ }^{19}$ We were not able to replicate those results, which could be due to our use of different immunohistochemical methods, tissue microarray instead of whole tissue sections, or image analysis instead of microscope observation for assessment of immunostaining. MMP-9 immunohistochemical expression has been found to be associated with prognosis in some studies ${ }^{21,41,44}$ but not in others. ${ }^{23,44}$ We already reported that integrin- $\alpha \mathrm{v} \beta 6$ immunohistochemical overexpression was associated with poor overall survival in patients with adenocarcinoma and squamous cell carcinoma of the lung. ${ }^{20}$

Although epithelial-to-mesenchymal transition has been associated with activation of the EGFR 
pathway, ${ }^{9,22,41,45,46}$ we found no relationship between the expression of these markers and EGFR immunohistochemical expression or the EGFR mutation status of tumors. Of interest, an epithelial-to-mesenchymal transition gene expression signature was identified in lung cancer cell lines that are sensitive to in vitro exposure to the EGFR tyrosine kinase inhibitor erlotinib. ${ }^{9}$ Moreover, the reexpression of E-cadherin has been shown to increase the sensitivity of lung cancer cell lines to EGFR tyrosine kinase inhibitors. ${ }^{47}$ Although a few studies have shown that E-cadherin overexpression of lung cancer tumors determines sensitivity to EGFR inhibitor, ${ }^{9}$ no association was found between EGFR mutation and E-cadherin expression in one study. $^{48}$

Lung cancer is believed to develop from a series of preneoplastic lesions in the respiratory mucosa, and these abnormalities are frequently extensive and multifocal throughout the respiratory epithelium, indicating a field effect or field cancerization phenomenon. ${ }^{5}$ These histopathologic changes have been well defined for squamous cell carcinoma, but they are poorly defined for adenocarcinoma. ${ }^{5}$ Mucosal changes in the large airways that may precede invasive squamous cell carcinoma include squamous dysplasia and carcinoma in situ in the central bronchial airway. ${ }^{5}$ In these epithelial changes, we found increased mesenchymal phenotype levels (especially reduced E-cadherin and increased $\mathrm{N}$-cadherin and integrin- $\alpha \mathrm{v} \beta 6$ expression), and these levels corresponded with increased histopathologic severity. These findings indicate that epithelial-to-mesenchymal transition occurs early in the pathogenesis of lung cancer. To our knowledge, only reduced E-cadherin expression has been previously described by us ${ }^{49}$ and others. ${ }^{50}$ to commence at the squamous dysplasia stage. Of the five markers studied, membrane integrin $-\alpha \mathrm{v} \beta 6$ had the clearest stepwise increase in expression, which corresponded to histopathologic severity (from normal and reactive bronchial epithelia to squamous dysplasia and invasive squamous cell carcinoma). Thus, integrin- $\alpha \mathrm{v} \beta 6$ may be a novel marker for monitoring the progression of centrally located preneoplastic lesions in smokers.

Because of their overexpression in malignant cells and their role in cell survival, motility, and invasion, several proteins related to epithelial-tomesenchymal transition, including $\mathrm{N}$-cadherin and integrins, have been considered as therapeutic targets in cancer. ${ }^{37,51}$ However, the expression of these proteins in the spectrum of advanced lung tumors, including metastatic sites, has not been previously studied. In addition, to our knowledge, this is the first study to assess the expression of these transition markers in lung cancer tissue specimens obtained from metastatic sites. It has been suggested that the progression of epithelial tumors involves spatial and temporal occurrences of mesenchymal transition, whereby tumor cells acquire a more invasive and metastatic phenotype. ${ }^{6,8}$ The disseminated mesenchymal tumor cells must then undergo a reversed mesenchymal-to-epithelial transition at the site of metastasis because metastasis recapitulates the pathologic characteristics of the cells' corresponding primary tumors. Interestingly, we found lower mesenchymal phenotype levels in lung cancer brain metastases than in primary tumors, confirming that cellular plasticity, which allows cells to undergo either transition in the appropriate microenvironments, is important in metastasis. This finding emphasizes the importance of characterizing and understanding the molecular events in lung cancer metastasis.

In summary, to our knowledge, this study is the first to comprehensively evaluate the expression of five proteins related to epithelial-to-mesenchymal transition phenotype throughout the early development and progression of lung cancer. We demonstrated that this phenotype is frequently expressed in primary squamous cell carcinoma and adenocarcinoma of the lung; and this process is an early phenomenon in the pathogenesis of squamous cell carcinoma, and lung tumor brain metastases show characteristics of mesenchymal-to-epithelial transition.

\section{Acknowledgements}

This study was supported in part by a grant from the Specialized Program of Research Excellence (SPORE) in Lung Cancer grant P50 CA70907 (IIW) and grant 1RO1CA106646 (KCB), National Cancer Institute, Bethesda, MD, and Department of Defense grant W81XWH-04-1-0142 (IIW).

\section{References}

1 Jemal A, Siegel R, Ward E, et al. Cancer statistics, 2008. CA Cancer J Clin 2008;58:71-96.

2 Minna JD, Gazdar A. Focus on lung cancer. Cancer Cell 2002;1:49-52.

3 Travis WD, Brambilla E, Muller-Hermelink HK, et al. Tumours of the lung In: Travis WD, Brambilla E, Muller-Hermelink HK, Harris CC (eds). Pathology and Genetics: Tumours of the Lung, Pleura, Thymus and Heart, Vol. 10, 10th edn. International Agency for Research on Cancer (IARC): Lyon, 2004, pp 9-124.

4 van Zandwijk N. Neoadjuvant strategies for nonsmall cell lung cancer. Lung Cancer 2001;34(Suppl 2): S145-S150.

5 Wistuba II. Genetics of preneoplasia: lessons from lung cancer. Curr Mol Med 2007;7:3-14.

6 Thiery JP. Epithelial-mesenchymal transitions in tumour progression. Nat Rev Cancer 2002;2:442-454.

7 Thiery JP, Sleeman JP. Complex networks orchestrate epithelial-mesenchymal transitions. Nat Rev Mol Cell Biol 2006;7:131-142.

8 Hugo H, Ackland ML, Blick T, et al. Epithelialmesenchymal and mesenchymal-epithelial transitions 
in carcinoma progression. J Cell Physiol 2007;213: 374-383.

9 Yauch RL, Januario T, Eberhard DA, et al. Epithelial versus mesenchymal phenotype determines in vitro sensitivity and predicts clinical activity of erlotinib in lung cancer patients. Clin Cancer Res 2005;11: 8686-8698.

10 Bremnes RM, Veve R, Hirsch FR, et al. The E-cadherin cell-cell adhesion complex and lung cancer invasion, metastasis, and prognosis. Lung Cancer 2002;36: 115-124.

11 Thomson S, Buck E, Petti F, et al. Epithelial to mesenchymal transition is a determinant of sensitivity of non-small-cell lung carcinoma cell lines and xenografts to epidermal growth factor receptor inhibition. Cancer Res 2005;65:9455-9462.

12 Mountain CF. Revisions in the International System for Staging Lung Cancer. Chest 1997;111:1710-1717.

13 Elayadi AN, Samli KN, Prudkin L, et al. A peptide selected by biopanning identifies the integrin alphavbeta6 as a prognostic biomarker for nonsmall cell lung cancer. Cancer Res 2007;67:5889-5895.

14 Wolff AC, Hammond ME, Schwartz JN, et al. American Society of Clinical Oncology/College of American Pathologists guideline recommendations for human epidermal growth factor receptor 2 testing in breast cancer. J Clin Oncol 2007;25:118-145.

15 Shigematsu H, Lin L, Takahashi T, et al. Clinical and biological features associated with epidermal growth factor receptor gene mutations in lung cancers. J Natl Cancer Inst 2005;97:339-346.

16 Tang X, Shigematsu H, Bekele BN, et al. EGFR tyrosine kinase domain mutations are detected in histologically normal respiratory epithelium in lung cancer patients. Cancer Res 2005;65:7568-7572.

17 Kase S, Sugio K, Yamazaki K, et al. Expression of E-cadherin and beta-catenin in human non-small cell lung cancer and the clinical significance. Clin Cancer Res 2000;6:4789-4796.

18 Deeb G, Wang J, Ramnath N, et al. Altered E-cadherin and epidermal growth factor receptor expressions are associated with patient survival in lung cancer: a study utilizing high-density tissue microarray and immunohistochemistry. Mod Pathol 2004;17:430-439.

19 Nakashima T, Huang C, Liu D, et al. Neural-cadherin expression associated with angiogenesis in non-smallcell lung cancer patients. Br J Cancer 2003;88: 1727-1733.

20 Elayadi AN, Samli KN, Prudkin L, et al. A peptide selected by biopanning identifies the integrin \{alpha\}v\{beta\}6 as a prognostic biomarker for nonsmall cell lung cancer. Cancer Res 2007;67:5889-5895.

21 Cox G, Jones JL, O’Byrne KJ. Matrix metalloproteinase 9 and the epidermal growth factor signal pathway in operable non-small cell lung cancer. Clin Cancer Res 2000;6:2349-2355.

22 Swinson DE, Cox G, O’Byrne KJ. Coexpression of epidermal growth factor receptor with related factors is associated with a poor prognosis in non-small-cell lung cancer. Br J Cancer 2004;91:1301-1307.

23 Leinonen T, Pirinen R, Bohm J, et al. Expression of matrix metalloproteinases 7 and 9 in non-small cell lung cancer. Relation to clinicopathological factors, beta-catenin and prognosis. Lung Cancer 2006;51:313-321.

24 Thompson EW, Newgreen DF, Tarin D. Carcinoma invasion and metastasis: a role for epithelial-mesenchymal transition? Cancer Res 2005;65:5991-5995; discussion 5.
25 Bates RC. The alphaVbeta6 integrin as a novel molecular target for colorectal cancer. Future Oncol 2005;1:821-828.

26 Peinado H, Portillo F, Cano A. Transcriptional regulation of cadherins during development and carcinogenesis. Int J Dev Biol 2004;48:365-375.

27 Tomita K, van Bokhoven A, van Leenders GJ, et al. Cadherin switching in human prostate cancer progression. Cancer Res 2000;60:3650-3654.

28 Gilles C, Polette M, Piette J, et al. Vimentin expression in cervical carcinomas: association with invasive and migratory potential. J Pathol 1996;180:175-180.

29 Charalabopoulos K, Gogali A, Kostoula OK, et al. Cadherin superfamily of adhesion molecules in primary lung cancer. Exp Oncol 2004;26:256-260.

30 Shintani Y, Maeda M, Chaika N, et al. Collagen I promotes epithelial-to-mesenchymal transition in lung cancer cells via transforming growth factor-beta signaling. Am J Respir Cell Mol Biol 2008;38:95-104.

31 Shih JY, Tsai MF, Chang TH, et al. Transcription repressor slug promotes carcinoma invasion and predicts outcome of patients with lung adenocarcinoma. Clin Cancer Res 2005;11:8070-8078.

32 Dohadwala M, Yang SC, Luo J, et al. Cyclooxygenase-2dependent regulation of E-cadherin: prostaglandin $\mathrm{E}(2)$ induces transcriptional repressors ZEB1 and snail in non-small cell lung cancer. Cancer Res 2006;66:5338-5345.

33 Schutz A, Schneidenbach D, Aust G, et al. Differential expression and activity status of MMP-1, MMP-2 and MMP-9 in tumor and stromal cells of squamous cell carcinomas of the lung. Tumour Biol 2002;23:179-184.

34 Illman SA, Lehti K, Keski-Oja J, et al. Epilysin (MMP-28) induces TGF-beta mediated epithelial to mesenchymal transition in lung carcinoma cells. J Cell Sci 2006;119:3856-3865.

35 Zochbauer-Muller S, Fong KM, Virmani AK, et al. Aberrant promoter methylation of multiple genes in non-small cell lung cancers. Cancer Res 2001; 61:249-255.

36 Cavallaro U, Christofori G. Cell adhesion and signalling by cadherins and Ig-CAMs in cancer. Nat Rev Cancer 2004;4:118-132.

37 Eble JA, Haier J. Integrins in cancer treatment. Curr Cancer Drug Targets 2006;6:89-105.

38 Ahmed N, Thompson EW, Quinn MA. Epithelialmesenchymal interconversions in normal ovarian surface epithelium and ovarian carcinomas: an exception to the norm. J Cell Physiol 2007;213:581-588.

39 Regezi JA, Ramos DM, Pytela R, et al. Tenascin and beta 6 integrin are overexpressed in floor of mouth in situ carcinomas and invasive squamous cell carcinomas. Oral Oncol 2002;38:332-336.

40 Friedl P, Wolf K. Tumour-cell invasion and migration: diversity and escape mechanisms. Nat Rev Cancer 2003;3:362-374

41 Ferrigan L, Wallace WA. Predicting non-small cell lung cancer expression of epidermal growth factor receptor and matrix metalloproteinase 9 from immunohistochemical staining of diagnostic biopsy samples. Eur J Cancer 2004;40:1589-1592.

42 Liu D, Huang C, Kameyama K, et al. E-cadherin expression associated with differentiation and prognosis in patients with non-small cell lung cancer. Ann Thorac Surg 2001;71:949-954; discussion 54-5.

43 Choi YS, Shim YM, Kim SH, et al. Prognostic significance of E-cadherin and beta-catenin in resected 
stage I non-small cell lung cancer. Eur J Cardiothorac Surg 2003;24:441-449.

44 Iniesta P, Moran A, De Juan C, et al. Biological and clinical significance of MMP-2, MMP-9, TIMP-1 and TIMP-2 in non-small cell lung cancer. Oncol Rep 2007;17:217-223.

45 Fedor-Chaiken M, Hein PW, Stewart JC, et al. E-cadherin binding modulates EGF receptor activation. Cell Commun Adhes 2003;10:105-118.

46 Andl CD, Rustgi AK. No one-way street: cross-talk between e-cadherin and receptor tyrosine kinase (RTK) signaling: a mechanism to regulate RTK activity. Cancer Biol Ther 2005;4:28-31.

47 Witta SE, Gemmill RM, Hirsch FR, et al. Restoring Ecadherin expression increases sensitivity to epidermal growth factor receptor inhibitors in lung cancer cell lines. Cancer Res 2006;66:944-950.
48 Miyanaga A, Gemma A, Ando M, et al. E-cadherin expression and epidermal growth factor receptor mutation status predict outcome in non-small cell lung cancer patients treated with gefitinib. Oncol Rep 2008;19:377-383.

49 Shen J, Behrens C, Wistuba II, et al. Identification and validation of differences in protein levels in normal, premalignant, and malignant lung cells and tissues using high-throughput Western Array and immunohistochemistry. Cancer Res 2006;66:11194-11206.

50 Kato Y, Hirano T, Yoshida K, et al. Frequent loss of Ecadherin and/or catenins in intrabronchial lesions during carcinogenesis of the bronchial epithelium. Lung Cancer 2005;48:323-330.

51 Mariotti A, Perotti A, Sessa C, et al. N-cadherin as a therapeutic target in cancer. Expert Opin Investig Drugs 2007;16:451-465. 